Check for updates

Cite this: RSC Adv., 2018, 8, 26036

\title{
Fabrication of 3D conductive circuits: print quality evaluation of a direct ink writing process
}

\author{
F. Tricot, (D) *a C. Venet, ${ }^{\text {b }}$ D. Beneventi, ${ }^{a}$ D. Curtil, ${ }^{a}$ D. Chaussy, ${ }^{a}$ T. P. Vuong, ${ }^{c}$ \\ J. E. Broquin ${ }^{c}$ and N. Reverdy-Bruas ${ }^{a}$
}

The use of conductive inks and direct writing techniques for the fabrication of electronic circuits on complex substrates is attracting ever increasing interest. However, the existing knowledge is only focused on the electrical performances of the produced smart objects with no direct correlation with the conductive paths morphology and printing conditions. In order to evaluate the printing quality of a direct writing process using an eccentric screw dispenser, a printing device for the deposition of silver paste on 3D objects was developed. Lines of different widths were printed on flat PC + ABS substrates by means of the developed printing device and a conventional screen printing process. The developed process permitted printing lines as thin as possible with screen printing but with a better regularity of their edges. However unlike screen printed lines, the thickness of the lines was dependent on their width. Finally, the possibility to print on 3D objects was demonstrated.

Received 19th April 2018

Accepted 10th July 2018

DOI: $10.1039 / \mathrm{c} 8 \mathrm{ra03380c}$

rsc.li/rsc-advances

based on the injection-moulding of a thermoplastic charged

\section{Introduction}

As a consequence of the lightning development of the Internet and network technologies over the past few decades, the Internet of Things (IoT), which deals with the interconnection of devices and their interactions with humans, is a hot topic for modern society. ${ }^{1}$ There is a drive for new capabilities, like sensors, actuators, and communication functions, to be incorporated in daily objects. This is leading to the development of new technologies combining mechanical and electronical functions to produce interconnected systems. ${ }^{2}$ One such system that is increasingly proving to be attractive in several fields, such as automotive, ${ }^{3,4}$ medical ${ }^{5,6}$ and telecommunications, ${ }^{7,8}$ is three-dimensional moulded interconnected devices (3D-MID). ${ }^{9}$ This requires additional steps in the fabrication of thermoplastic objects to incorporate electrical components in or at the surface of the object. To date, two processes are industrially available. They both need specific thermoplastics and chemical treatments for metallizing, which requires substantive investment. ${ }^{10}$ On the one hand, the two-shot moulding consists of two successive injection mouldings, one of a non-plateable thermoplastic to constitute the object and one of a plateable thermoplastic to pre-form the electric elements. ${ }^{11}$ On the other hand, the second technique involves laser direct structuring (LDS), developed by LPKF Laser \& Electronics AG, which is

\footnotetext{
${ }^{a}$ Institute of Engineering, Univ. Grenoble Alpes, CNRS, Grenoble INP, LGP2, F-38000 Grenoble, France. E-mail: fanny.tricot@grenoble-inp.fr

${ }^{b}$ Schneider Electric, France
}

'Institute of Engineering, Univ. Grenoble Alpes, CNRS, Grenoble INP, IMEP LAHC, F38000 Grenoble, France with metallic precursors. Once the object has been patterned by moulding, a laser is used to selectively activate the conductive areas. ${ }^{3}$ Finally, for both processes, a chemical metallization is performed to achieve the fabrication of electric elements. ${ }^{10}$

To propose an alternative to both two-shot moulding and LDS techniques, some research groups have focused on additive manufacturing thanks to the emergence of 3D printing techniques. The latter offers indeed polyvalent and cost-effective solutions since it does not require specific materials and allows avoiding chemical metallization. Several articles report the adjustment of low $\operatorname{cost}^{12,13}$ or home-made ${ }^{14,15} 3 \mathrm{D}$ printers based on a fused deposition modelling (FDM) process. The printing substrate can be a thermoplastic product, ${ }^{12-14,16}$ a wafer ${ }^{17}$ or a glass slide. ${ }^{17}$ It can be either previously made by other techniques ${ }^{14,17}$ or produced within the FDM process ${ }^{12,13}$ as the conductive elements are deposited. The latter are fabricated by the selective coating of conductive inks, mostly silver paste. ${ }^{14,16,17}$ To do this, the FDM extruder is usually replaced by a dispenser composed of a syringe filled with the ink. The conductive material is pushed through a needle to be ejected thanks to the air pressure. ${ }^{14,15,17}$ One article reports the use of a dispenser composed of an eccentric screw driving the ink towards the needle. ${ }^{18}$ That fabrication process is mainly used to incorporate a wireless communication function to the objects..$^{12,13,16}$ In all readings, the evaluation of the fabricated product is considered from an electrical and communicating performance point of view.

This paper proposes to evaluate the deposition process inspired by FDM 3D printing as a printing process. The aim was to discuss the quality of the printing made by the 3D writing 
process on a thermoplastic substrate commonly used for the manufacturing of daily objects in comparison with the printing quality obtained by a conventional printing techniques (i.e. screen printing).

To set up the study, a commercial screen printing silver ink was printed according to a lines design on 2D PC $+\mathrm{ABS}$ substrates by two means: the standard screen printing process and the developed direct ink writing method. The ink behaviour during the two processes was first considered. Then, the characteristics of the printed lines were determined and compared.

\section{Experimental}

\section{Materials}

Substrates on which the prints were performed were supplied by Schneider Electric SA in the form of $80 \times 80 \times 3 \mathrm{~mm}^{3} \mathrm{PC}+\mathrm{ABS}$ thermoplastic plates with a surface roughness $R_{\mathrm{a}}$ of $643 \pm$ $20 \mathrm{~nm}$ (as determined with an Alicona InfiniteFocus optical profilometer). This type of thermoplastic material, which cannot withstand temperatures higher than $110{ }^{\circ} \mathrm{C}$, was selected because it is abundantly used in consumer goods manufacturing. Because of these substrate characteristics, the main properties considered to choose the conductive ink were a drying temperature inferior to $110^{\circ} \mathrm{C}$ and a compatibility with solvent-sensitive substrates. The ability of printing ultrafine lines was not taken into account. The silver ink Electrodag 418SS from Henkel was used since it satisfied the substrate requirements. According to the technical data sheet, an ink layer of $25 \mu \mathrm{m}$ leads to a resistivity inferior to $750 \mathrm{n} \Omega \mathrm{m}$ and the ink solid content is $66.5 \%$ with a silver particle size D50 lower than $8 \mu \mathrm{m}$ and D95 below $24 \mu \mathrm{m}$. No treatment was done on the surface of the substrates before printing.

\section{Printing}

Screen printing process. The screen printing technique was used as the reference printing process. Screen printing is a push-through process, where the ink passes through a screen to be deposited on the substrate. The screen is made of a fabric, where some meshes are selectively coated by a photopolymer film so that the remaining open meshes constitute the pattern to print. Printing was realized with the DEK Horizon 03iX Plateform. The screen was produced using a polyester fabric with a mesh of 100 threads per $\mathrm{cm}$, a thread having a diameter of 40 $\mu \mathrm{m}$ and a capillary film of $30 \mu \mathrm{m}$ thick (i.e. according to the recommendations for the Henkel ink). The printing parameters were optimized according to the Henkel ink, the PC + ABS substrate and the produced screen. The selected parameters were the following:

- Squeegee pressure: $7 \mathrm{~kg}$ for a squeegee (70-75 shore) of $350 \mathrm{~mm}$ length.

- Squeegee speed: $10 \mathrm{~mm} \mathrm{~s}^{-1}$.

- Off contact: $4 \mathrm{~mm}$.

After printing, the lines were dried at $110{ }^{\circ} \mathrm{C}$ for $20 \mathrm{~min}$.

Eccentric screw dispenser on $3 \mathrm{D}$ printer. A device adapted to perform printing on 3D objects was set up involving the combination of two apparatuses:
(1) A Cartesian 3D printer Prusa i3 rework 1.5 (EmotionTech) controlled by an Arduino Mega 2560/Ramps 1.4 board and Repetier software.

(2) An accurate volumetric dosing system (Ecopen 300 Preeflow provided by Viscotec). The device comprised two elements: the printing head, which looks like a pen, and a controller. In this system, the ink to print is stored in a pressurized external syringe and it is pneumatically fed into the printing head, which constitutes a soft polymeric stator and a stainless steel eccentric screw. The rotation of the endless screw brings the ink to the extremity of the stator, where a needle is fixed. The fluid is then pushed through the needle to reach the surface to print. The internal diameter $\varnothing_{\text {internalneedle }}(\mu \mathrm{m})$ of the used needle was chosen in accordance with the size of particles contained in the ink. The controller was linked at the Ecopen head by an electronic wired connection, which permits managing the ink flow rate $Q$

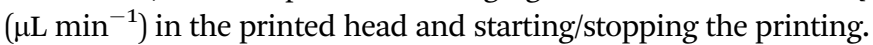

The two described devices were assembled to form the 3D printing process used in this study. For this, the original FDM printing head of the $3 \mathrm{D}$ printer was replaced by the Ecopen connected to its controller. An electronic wired connection was made between the Ecopen controller and the Arduino board in order to synchronise ink dispensing (on a start/stop basis) with the XYZ motion of the 3D printer. Fig. 1(a) presents a photograph of the device and Fig. 1(b) illustrates the ink route in the Ecopen printed head.

For all the studies, the distance between the needle and the surface to print $z_{\text {ecopen }}(\mu \mathrm{m})$ was fixed according to the internal diameter of the needle. Table 1 summarizes the parameters for which the effect of their variation on the printed pattern was studied.

The first study consisted in evaluating the effect of the fluid flow rate on the printed pattern. For that, the moving speed of the $3 \mathrm{D}$ printer $v\left(\mathrm{~mm} \mathrm{~min}^{-1}\right)$ was monitored to a level just inferior to the ink speed in the Ecopen. Besides, it had to be inferior to $1000 \mathrm{~mm} \mathrm{~min}^{-1}$ to avoid printing defects due to mechanical vibrations of the 3D printer. The average ink speed in the dispensing needle was estimated using eqn (1):

$$
v_{\text {ink in Ecopen }}=\frac{Q}{\pi R^{2}}
$$

where $v_{\text {ink in Ecopen }}\left(\mathrm{m} \mathrm{s}^{-1}\right)$ is the ink speed in the needle, $Q\left(\mathrm{~m}^{3}\right.$ $\left.\mathrm{s}^{-1}\right)$ is the fluid flow rate and $R(\mathrm{~m})$ is the inner diameter of the needle. The fluid flow rate was then fixed at its minimal value and the effect of increasing the moving speed was studied. Two needles of different inner diameters (i.e. 110 and $200 \mu \mathrm{m}$ ) were considered for each experiment.

After printing, the lines were dried at $110^{\circ} \mathrm{C}$ for $90 \mathrm{~min}$.

Printing patterns. Horizontal and vertical lines were realized. For the screen printing tests, the printing plate constituted a set of horizontal and vertical lines with a length of $30 \mathrm{~mm}$ and with widths decreasing as follows: $2 \mathrm{~mm}, 1 \mathrm{~mm}, 800 \mu \mathrm{m}, 500 \mu \mathrm{m}, 200$ $\mu \mathrm{m}, 100 \mu \mathrm{m}, 50 \mu \mathrm{m}$. Horizontal lines were lines parallel to the squeegee, whereas vertical lines were perpendicular to the squeegee and hence parallel to the displacement during printing.

With the developed printing device lines, the associated speed and dispensing on/off switch were both directly 
a)

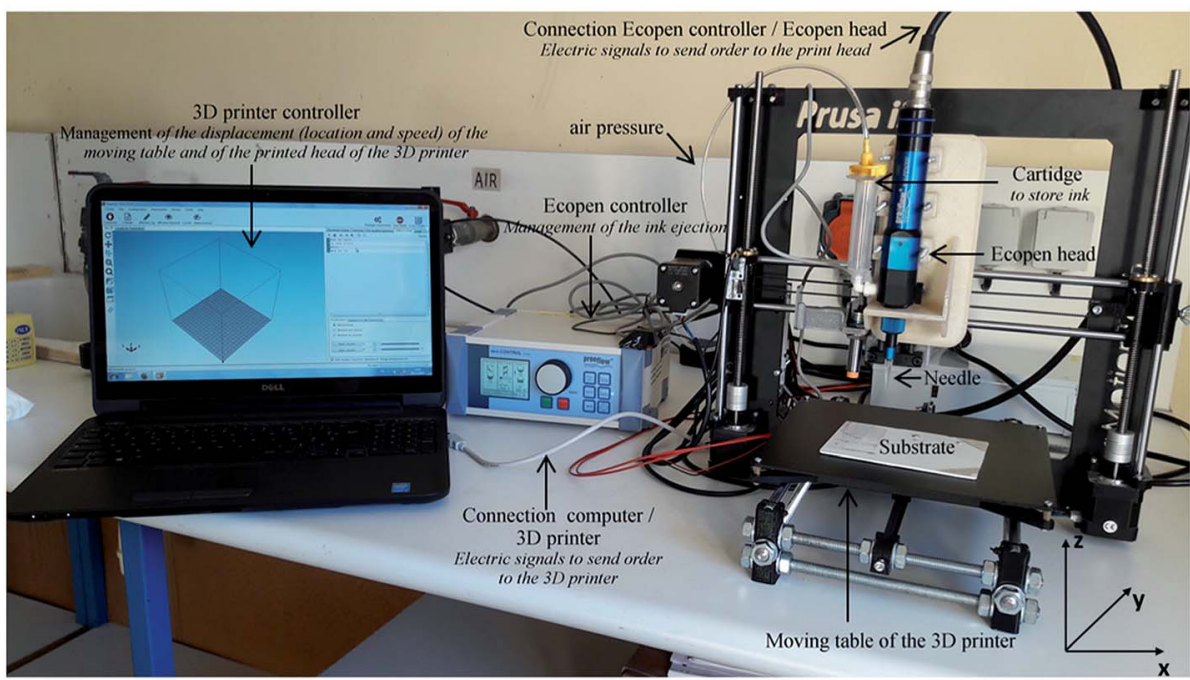

b)

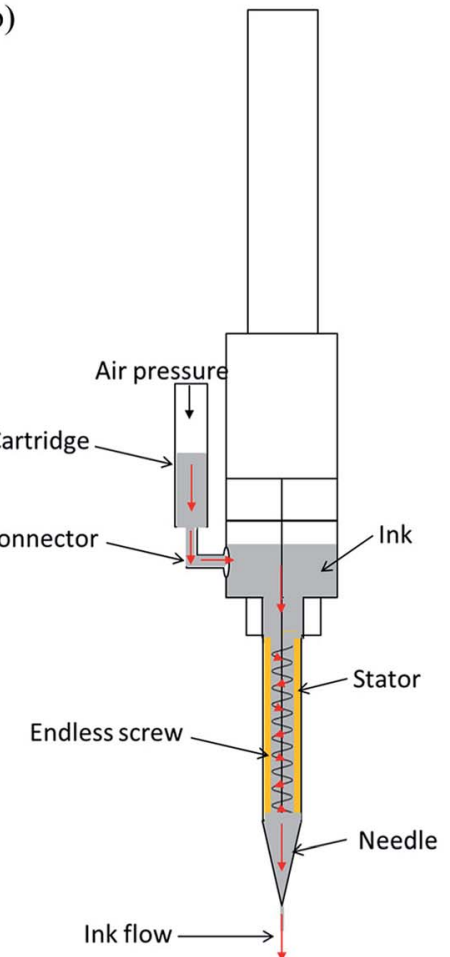

Fig. 1 (a) Photograph of the developed printing device, (b) schema of the Ecopen head and route of the ink flow. The ink is represented in grey colour. The route is represented by the red arrow.

programmed in g-code or drawn using CAD software and converted into a g-code using Repetier/Slicer.

\section{Characterizations}

Rheological properties. Rheological measurements were performed with the Anton Paar MCR-302 rheometer. A parallel plate geometry (PP25-25 mm) was chosen and a gap of $100 \mu \mathrm{m}$ was fixed. During the experiment, the temperature was set at $25{ }^{\circ} \mathrm{C}$. Before each measurement, a pre-treatment composed by a step at $100 \mathrm{~s}^{-1}$ during $60 \mathrm{~s}$ followed by a rest period of $300 \mathrm{~s}$ was applied in order to standardize the initial state of the ink. ${ }^{19,20}$

Rotational rheological experiments were performed to simulate the ink behaviour during the two studied printing processes.

On the one hand, the ink was submitted to the following successive shear rate values: $1 \mathrm{~s}^{-1}-150 \mathrm{~s} / 1000 \mathrm{~s}^{-1}-60 \mathrm{~s} / 1 \mathrm{~s}^{-1}-$ $150 \mathrm{~s}$. This procedure imitates the screen printing process. The first step corresponds to the ink being on the screen, the second step represents the passing of the ink through the screen during the squeegee stroke and the third step simulates the remaining ink being deposited on the substrate. ${ }^{19-21}$
On the other hand, the ink flow in the volumetric pump was simulated by four steps using the following assumptions:

- First, the passage of the ink in the eccentric screw was represented by a low shear rate of $1 \mathrm{~s}^{-1}$ during $150 \mathrm{~s}$.

- Second, the needle was assimilated to a tube and the ink behaviour was preliminary estimated by a power law. ${ }^{22}$ The passage of the ink through the needle was characterized by the maximal shear rate at the walls of the needle, given by eqn (2): ${ }^{22}$

$$
\tau_{\mathrm{w}}=K\left(\frac{3 n+1}{4 n} \times \frac{4 Q}{\pi R^{3}}\right)^{n}
$$

where $n$ and $K$ are determined by the power law, $Q$ is the applied fluid flow rate in the Ecopen and $R$ is the inner radius of the needle.

- Third, during the deposition of the ink on the substrate, the ink is supposed to be between a fixed plane (the substrate) and a mobile plane in translation (the needle). The shear rate was calculated using eqn (3):

$$
\dot{\gamma}=\frac{v}{z_{\text {ecopen }}}
$$

where $\dot{\gamma}\left(\mathrm{s}^{-1}\right)$ is the shear rate and $v\left(\mathrm{~m} \mathrm{~s}^{-1}\right)$ and $z_{\text {ecopen }}(\mathrm{m})$ are the parameters used during the experiment.

Table 1 Studied and fixed parameters of the device adapted from a 3D printer

\begin{tabular}{lllll}
\hline Parameters & $Q\left(\mu \mathrm{L} \mathrm{\operatorname {min } ^ { - 1 } )}\right.$ & $V\left(\mathrm{~mm} \mathrm{~min}^{-1}\right)$ & $z_{\text {ecopen }}(\mu \mathrm{m})$ & $\emptyset_{\text {internalneedle }}(\mu \mathrm{m})$ \\
\hline Values & $5-100$ & $150-1000$ & $=\varnothing_{\text {internalneedle }}$ & $110 \& 200$
\end{tabular}


- Fourth, a period of rest was imposed with a shear rate at 1 $\mathrm{s}^{-1}$ during $150 \mathrm{~s}$.

Oscillatory measurements were also carried out to characterize the viscoelastic properties of the ink. A frequency of $1 \mathrm{~Hz}$ was selected. Beforehand, the linear viscoelastic area (LVE) was determined. This represents the region where the fluid displays a solid viscoelastic behaviour. ${ }^{23}$ Here, the stress is too weak to break particles network and the ink behaves as a hooked spring. A procedure representative of the screen printing process was applied. It was composed of three steps. During the first and the third, an oscillatory stress of $0.1 \mathrm{~Pa}$, including in the LVE area, was applied during $150 \mathrm{~s}$. This simulated the deposition of the ink on the screen before the squeegee stroke and the ink deposited on the substrate after printing. The second step was characterized by an applied oscillatory stress (3000 Pa) beyond the LVE area in order to simulate the breaking of the ink structure during the squeegee stroke. The phase angle $\delta\left(^{\circ}\right)$ was measured during the oscillatory experiment. This represents the phase difference between the applied sinusoidal stress and the response of the fluid and indicates the predominant behaviour, elastic, or viscous, since its tangent is equal to the quotient of the loss modulus on the storage modulus. ${ }^{24,25}$ Here, a phase angle of $0^{\circ}$ corresponds to a solid material, whereas a value of $90^{\circ}$ is a characteristic of a liquid. ${ }^{19,20}$

All the measurements were repeated at least twice. For each measurement, a new sample of ink was considered.

Printed lines characterizations. Printed lines were characterised thanks to optical microscopic observations with a Zeiss Axio Imager (magnification $\times 5$, observation in bright and dark fields) and software based on Python language. The quality of lines was estimated by determining the variation of the edges of the printed lines compared to the straight edges of an ideal line. The software calculated the ideal straight upper and lower edges corresponding to the printed line. Then for each pixel on the edges of the printed line, the standard deviation between its position and the position of the corresponding pixel on the ideal edge was calculated. Finally the average of the calculated standard deviation for each edge was deduced. The results were presented on a graph representing the average standard deviation of each edge of the printed line according to the printing conditions. The width of printed lines was obtained by calculating the distance between the upper edge and the lower edge pixel by pixel. The average widths are reported in the study. Fig. 2 illustrates the procedure. For each printing condition, three printed lines were analyzed. For each line, six to eight segments of $2.7 \mathrm{~mm}$ length were analyzed by the developed software.

The thickness and the roughness of the printed lines were measured using an Alicona InfiniteFocus optical profilometer (magnification $\times 10$ and $\times 50$ ). Roughness measurements followed the ISO 4288 standard. For each printing condition, 15 to 25 thickness and roughness measurements were done. The adhesion of lines on the substrate was evaluated with the tape test, following the ISO 2409-2007 2013 standard.

Evaluation of the printed lines' electrical resistivity. After drying, the electrical resistance $R$ of $30 \mathrm{~mm}$ length lines printed by screen printing and $60 \mathrm{~mm}$ length lines obtained with the

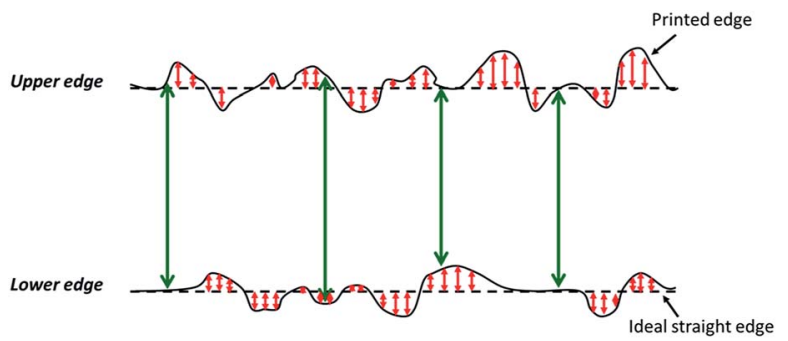

Fig. 2 Drawing of the top view of a printed line (continuous line) and the corresponding ideal line (dashed line). The red arrow represents the standard deviation calculated between the edge of the printed line and the edge of the corresponding ideal line. This calculation was made for each pixel on the printed edges. The green arrow represents the calculated width of the printed line. The calculation was made pixel by pixel.

direct ink writing process was measured. The resistivity $\rho(\mathrm{n} \Omega$ $\mathrm{m})$ of the lines was calculated from the measured width $w(\mathrm{~m})$, thickness $e(\mathrm{~m})$, length $L(\mathrm{~m})$ and resistance $R(\mathrm{n} \Omega)$ according to eqn (4):

$$
\rho=R \times \frac{w \times e}{L}
$$

\section{Results and discussion}

\section{Ink rheological behaviour during the printing processes}

Rotational experiment. The viscosity behaviour of the ink during the two printing processes was simulated and the results are presented in Fig. 3. For both printing techniques, the viscosity behaviour was similar, attesting to the compatibility of the screen printing ink and the $3 \mathrm{D}$ printing process. The ink exhibited a shear thinning behaviour typical of screen printing inks. ${ }^{19-21,26}$ The viscosity of the Henkel ink was lowered from $70 \%$ to $90 \%$ of its initial value according to the value of the applied shear rate. This behaviour ensures the flow of the ink through the screen during screen printing. As for the modified $3 \mathrm{D}$ printing, the highest shear rate, corresponding to the passing of the ink through the needle, facilitates the ink flow at

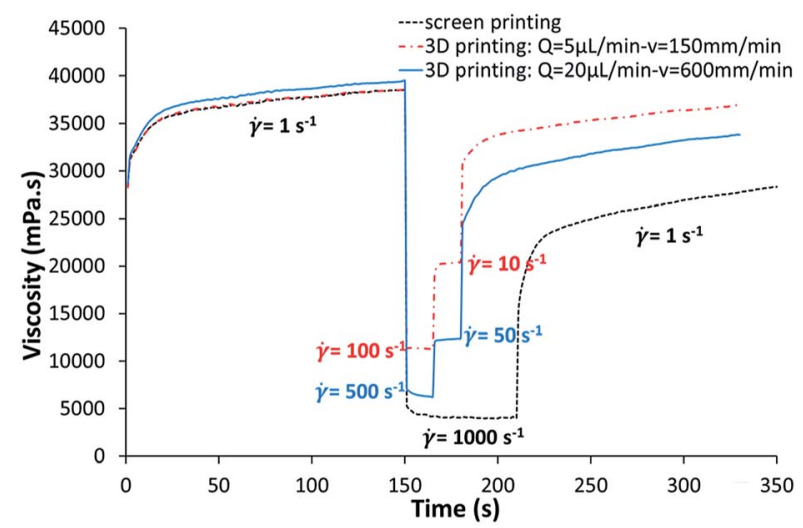

Fig. 3 Rheological behaviour of the Henkel ink during the simulation of printing processes. 
this step. The increase of viscosity during the next step, the deposition of the ink on the substrate, permits preserving the printed pattern. The behaviour of the ink is therefore compatible with the two studied printing processes.

Regardless of the specific process simulation, $150 \mathrm{~s}$ after printing, the ink recovered a lower viscosity value than the initial one, depending on the applied maximal shear rate. Here, $95 \%$ of the viscosity value was recovered after a succession of shearing at 100 then $10 \mathrm{~s}^{-1}$, whereas only $73 \%$ was reached after shearing at $1000 \mathrm{~s}^{-1}$. Consequently, the ink tended to spread on the substrate after printing. Because of that behaviour, the studied ink was not suitable for printing fine lines. ${ }^{27}$ The spreading coefficient appeared to be dependent on the intensity of the shearing and has to be taken into account during the determination of the printing parameters in order to respect the expected size of patterns.

Oscillatory experiments. Oscillatory experiments were also performed to evaluate the viscoelastic properties of the paste. The evolution of the phase angle during a screen printing simulation is given on Fig. 4. During the second step, involving the ink passing through the screen, the structure of the ink is totally broken down since the phase angle equals $90^{\circ}$. The material exhibits then a liquid behaviour as expected. ${ }^{\mathbf{1 9 2 0}}$ However, during the first and the last steps, the obtained phase angle is higher than $45^{\circ}$, meaning that the viscous contribution is predominant. This result disagrees with those already published in the literature. ${ }^{\mathbf{1 9 2 0}}$ A solid behaviour, without destructuring of the ink, is indeed expected during the deposition of the ink on the screen and the deposition of the ink on the substrate. The consequences of that behaviour of the Henkel ink are: (i) a facilitated ink flow during the squeegee stroke, since even in the rest period the Henkel ink tends to behave like a liquid and (ii) an ink spreading after the deposition on the substrate, leading to pattern widening. Considering the different steps of the ink deposition with the modified 3D printing process, the same viscous predominant behaviour entailing the same consequences could be expected.

Characteristics of the screen printed lines. Horizontal and vertical lines with different widths were printed using the screen printing process in order to produce a reference.

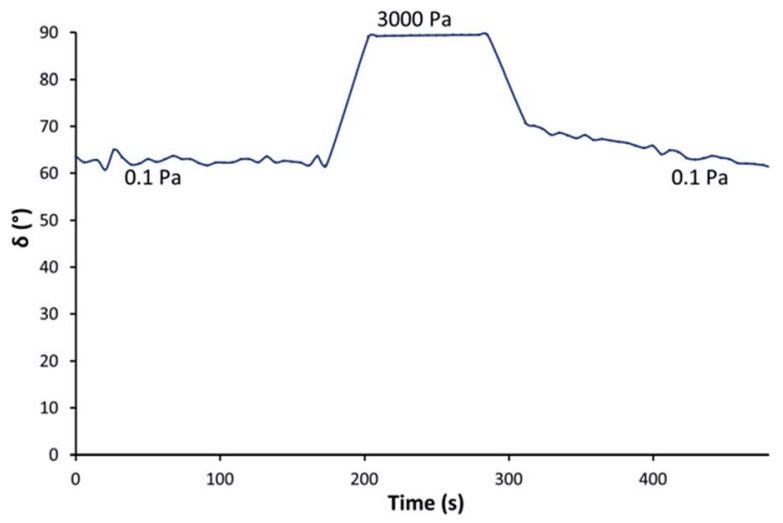

Fig. 4 Evolution of the phase angle $\delta$ during screen printing simulation in an oscillatory regime.
Lines continuity. First, the continuity of lines characterised by different theoretical width was studied. Microscopic observations are shown in Fig. 5 highlighting:

- Non-continuous lines for targeted widths of $50 \mu \mathrm{m}$.

- Continuous lines of $100 \mu \mathrm{m}$ theoretical width with some punctual lacks of ink.

- The presence of dribbles at the lower edge of some horizontal lines for targeted widths larger than $200 \mu \mathrm{m}$.

In the literature, some papers report the printing of continuous lines of $30^{28}$ and $50 \mu \mathrm{m}^{29-31}$ widths. The authors of those papers explain that to obtain these resolutions some precautions were necessary. Mainly, the ink rheology and the properties of the screen had to be adapted to fine lines. In this paper, on the one hand, the study of the ink rheological behaviour showed that it is not adapted to produce fine lines. On the other hand, the screen was produced according to the recommendation of the technical data sheet of the ink and not to fit with the properties that would permit achieving fine lines. As the emulsion thickness was $30 \mu \mathrm{m}$, the emulsion over mesh was higher than $10 \mu \mathrm{m}$, thus restricting the printing resolution. ${ }^{30}$ The diameter of threads constituting the fabric was about 40 $\mu \mathrm{m}$, while according to DEK recommendations, the minimal line width equals three times the thread diameter. Producing fine lines requires an advanced screen printing process. However, the objective of this paper was to demonstrate the ability of a new 3D printing process to print on a commonly used solvent-sensitive and poor thermal-resistant plastic substrate with a quality similar to that achieved by a standard screen printing process. Therefore, the printing of continuous fine lines was not in the scope of the present study.

The presence of dribbles at the lower edge of some horizontal continuous lines was attributed to the orientation of the lines with respect to the squeegee. This observation can be explained either by a screen deformation leading to a widening of the pattern or by the passing of a part of the ink below some threads of the fabric because of the complete liquid-like behaviour previously demonstrated. This last phenomenon was less observable on vertical lines because the ink spread below the fabric threads could be hidden by the line itself, which was perpendicular to the squeegee and hence parallel to its displacement. The production of repeatable and well-defined lines was therefore dependent on their orientation with respect to the squeegee.

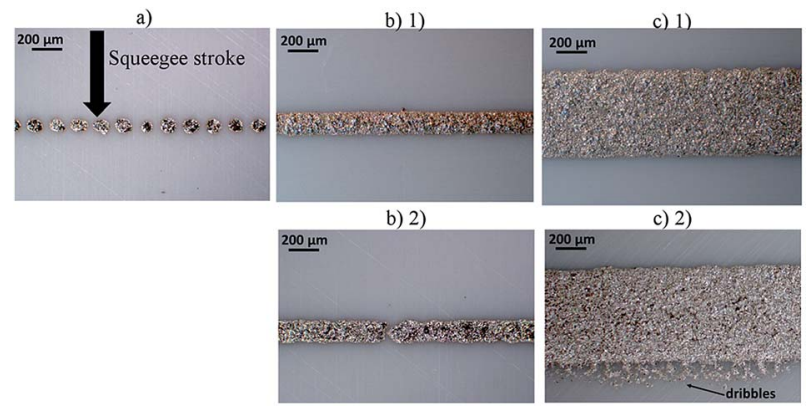

Fig. 5 Photographs of screen printed lines: (a) $50 \mu \mathrm{m}$ theoretical width line, (b) $100 \mu \mathrm{m}$ theoretical width line: (1) without defect, (2) with a lack of ink, (c) $500 \mu \mathrm{m}$ theoretical width line: (1) without defect and (c) (2) with dribbles at the lower edge. 
Some lines profiles were reconstructed from observations and measurements with the optical profilometer. An example of the reconstruction of a horizontal line is presented in Fig. 6 . According to the $3 \mathrm{D}$ view presented in Fig. 6(a), the line is visually regular and no significant defect is observable. The profile of the cross-section presented in Fig. 6(b) approaches a pulse function due to the principle of the screen printing process where the ink passes through opened meshes. The width and the thickness of the line were in agreement with the results presented in the following sections. Similar observations were done on other printed lines.

Line width. Dribbles aside, the deviation of the line width away from their targeted values were evaluated. Fig. 7 displays the average measured widths of sets of horizontal and vertical lines, each one characterized by one targeted width (dashed lines). The lines widening was slightly higher for horizontal lines than vertical lines due to the same phenomenon that causes dribbles on some horizontal lines. This phenomenon was more influential on fine lines. Table 2 highlights that a sensitive percentage had to be taken into account to anticipate the lines widening. Besides, for each targeted width, three lines were printed at different times and were analyzed. The measured widths were similar, exhibiting the reproducibility of the process. Finally, the small standard deviation associated with each calculated mean width indicated that the width of the lines remained constant all along the lines.

Edges regularity. The resolution of the lines was evaluated by determining the variations of the printed lines edges with respect to ideal straight edges. Fig. 8 presents the average standard deviation of edges for horizontal and vertical lines. The edges variations seem to be independent of the line width and there is no significant difference between the upper and lower edges. It can be noticed that the difference between the two edges of the two first horizontal $1000 \mu \mathrm{m}$ width lines (Fig. 8(a)) is attributed to the tendency of horizontal lines to present defects on their lower edge, as explained earlier. However, the line orientation could affect the edges variations since Fig. 8(b) globally presents higher and less reproducible standard deviations than Fig. 8(a). During the printing of vertical lines, the line edges are shaped all along the squeegee passing above the pattern design. The edge production is hence dependent on the movement of the squeegee. On the contrary, the printing of horizontal lines leads to the global constitution of each edge in one brief instant because of their collinear orientation with the squeegee action. Consequently, though

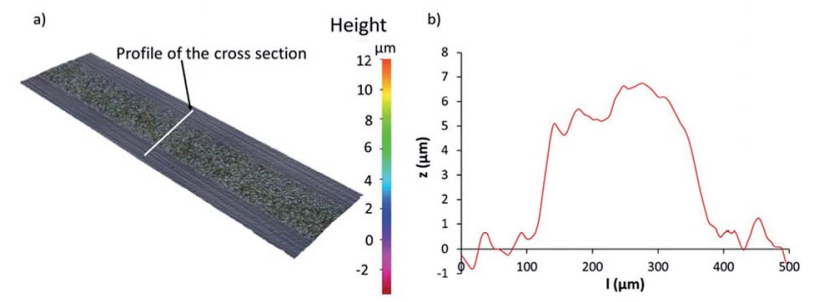

Fig. 6 (a) 3D view of a screen printed horizontal line with a theoretical width of $200 \mu \mathrm{m}$, (b) profile of the cross-section of the printed line presented in (a).

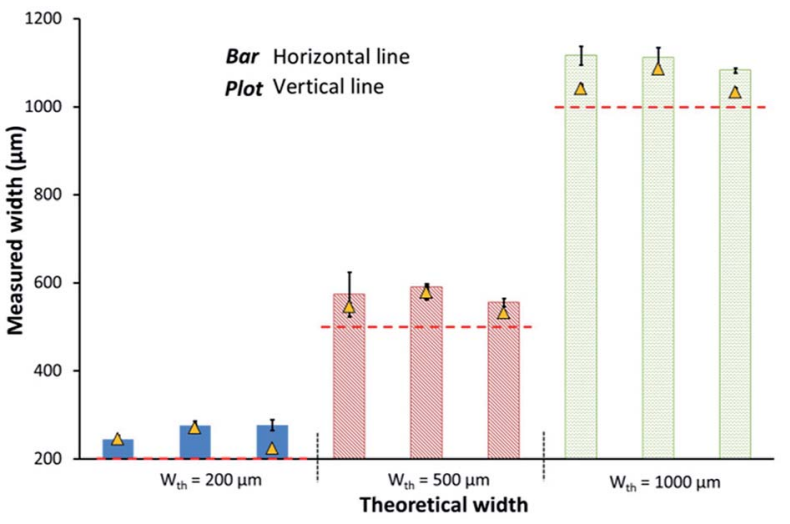

Fig. 7 Average widths of 3 printed lines/condition according to theoretical width values and the line orientation.

lines parallel to the squeegee during the printing are more prone to dribbles formation, if these defects are avoided, the horizontal line edges present the lowest variations.

For all the studied lines, the recorded standard deviations were lower than $17 \mu \mathrm{m}$. The highest amplitude of edge variations was therefore about $32 \mu \mathrm{m}$. The relevance of taking into account the highlighted edge variations was therefore dependent on the line width. Indeed, those variations represent $12 \%$ of the width for a $200 \mu \mathrm{m}$ targeted width, whereas they only correspond to less than $5 \%$ of the measured width of a line with a targeted width of $1000 \mu \mathrm{m}$.

Finally, the characterization of screen printed lines showed that:

- The spreading of the ink leads to a widening of the targeted width. The thinner the line, the stronger this effect is.

- The orientation of the line with respect to the squeegee impacts both the formation of dribbles at the lower edge and the regularity of line edges.

Other characteristics. The thickness, roughness and adhesion of printed lines were also measured. The thickness depended neither on the line width nor on the line orientation. For all the printed lines, it was equal to $5.1 \mu \mathrm{m} \pm 1.6 \mu \mathrm{m}$. The same observation was made for the roughness. Printed lines were characterized by a $R_{\mathrm{a}}$ value of $0.6 \mu \mathrm{m} \pm 0.1 \mu \mathrm{m}$. As for the adhesion of the printed lines on the PC + ABS substrate, during the tape test (ISO 2409-2007 2013 norm), no ink removal was observed.

\section{Characteristics of the lines printed with the $3 \mathrm{D}$ printing technique}

Using the screen printed lines as a reference, lines were drawn thanks to the volumetric dispenser fixed on the 3D printer. The printing parameters relative to the dispenser, i.e. the fluid flow rate $Q\left(\mu \mathrm{L} \mathrm{min}{ }^{-1}\right)$ and the inner diameter of the needle $\emptyset_{\text {needle }}$,

Table 2 Line widening converted to percentage of the targeted width

\begin{tabular}{llll}
\hline Targeted width $(\mu \mathrm{m})$ & $200 \mu \mathrm{m}$ & $500 \mu \mathrm{m}$ & $1000 \mu \mathrm{m}$ \\
\hline Widening $(\%)$ & $30 \%$ & $12 \%$ & $<10 \%$
\end{tabular}


a)

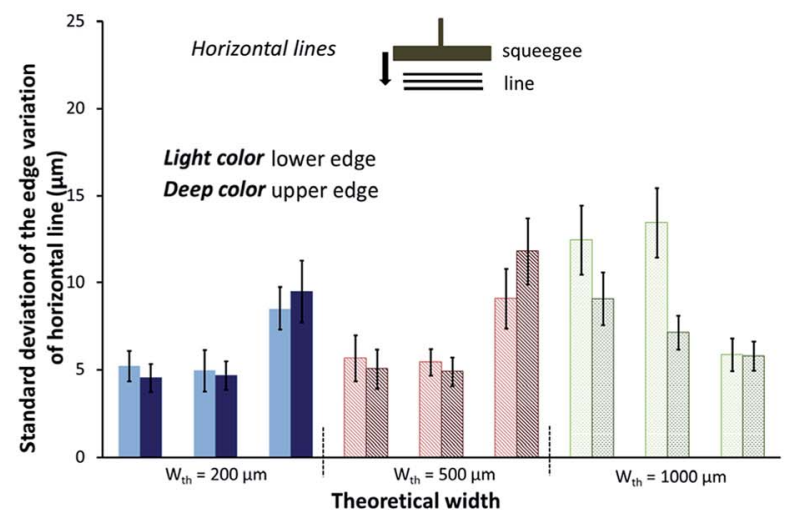

b)

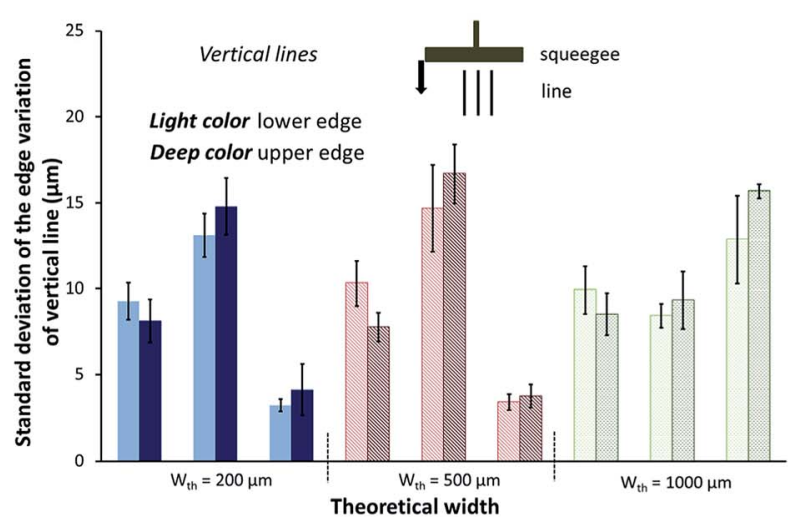

Fig. 8 Average standard deviation of the variation of the lower and upper edges of lines for targeted widths of 200, 500, and 1000 $\mu$ m: (a) 3 horizontal lines/conditions, (b) 3 vertical lines/conditions.

$(\mu \mathrm{m})$, and relative to the moving table, i.e. the moving speed $v$ $\left(\mathrm{mm} \min ^{-1}\right)$, were the parameters under investigation. The obtained lines were characterized and compared to the screen printing ones.

Shape of the lines. Printed lines were observed with the optical profilometer. Fig. 9 shows the 3D view and the profile of a cross-section of a line printed with a $200 \mu \mathrm{m}$ inner diameter needle, a fluid flow rate of $5 \mu \mathrm{L} \mathrm{min}{ }^{-1}$ and a table moving speed of $150 \mathrm{~mm} \mathrm{~min}^{-1}$. As for screen printed line, the 3D view did not highlight any visual defect and the line seemed to be regular. However, the profile of the line cross-section approached a Gaussian function, which is a major difference with what was observed for screen-printed lines. In fact, with the volumetric dispenser, the ink was actually deposited as a cord with more material at the centre than at the edges. This explains the observed shape for the line cross-section. The ink was concentrated at the centre of the line. The width and the thickness of the line were in good agreement with the results presented in the following sections.

Width and thickness of the lines. The printing parameters managed the width and the thickness of the printed lines, as can be observed in Fig. 10. In Fig. 10, graph (a) shows that lines with a width ranging from 245 and $1025 \mu \mathrm{m}$ were successfully produced. Widths lower than $350 \mu \mathrm{m}$ were obtained only when a needle of a $110 \mu \mathrm{m}$ inner diameter was used. Considering the line width, the $3 \mathrm{D}$ printing technique enable attaining the same result as from the screen printing process. One different point
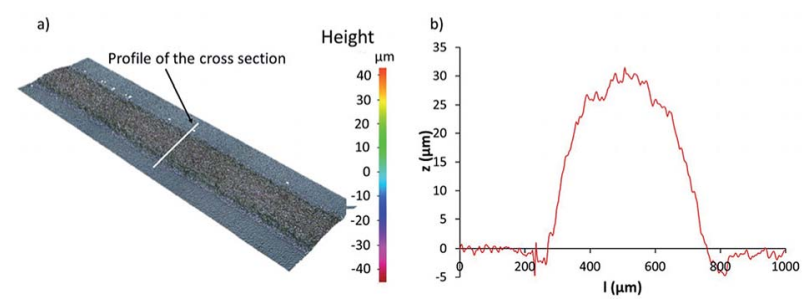

Fig. 9 (a) $3 d$ view of a line printed with the developed process with the following conditions: needle with a $200 \mu \mathrm{m}$ inner diameter, $Q=5$ $\mu \mathrm{L} \min ^{-1}, v=150 \mathrm{~mm} \mathrm{~min}^{-1}$, (b) profile of a cross-section of the line presented in (a). between the two processes, though, involves the thickness of the lines. Whereas it was constant for screen printed lines, graph (a) highlights the increase in thickness with the increase in width. The setting up of a higher fluid flow rate is necessary to increase the width, leading to an increase in the deposited quantity of ink and so to the augmentation of the thickness. The thinnest lines present a thickness similar to the one obtained with the screen printing technique.

According to Fig. 10(b), the vertical lines show a slightly higher width than horizontal lines for the same printing parameters. The orientation of lines only changes the part of the device that is moving. When printing vertical lines, the dispenser was motionless, while the substrate placed on the $3 \mathrm{D}$ printer table was moving. On the contrary, the dispenser was moving and the substrate was motionless for writing horizontal lines.

Otherwise, the reproducibility of the developed process when considering a $200 \mu \mathrm{m}$ inner diameter needle is highlighted in Fig. 10(b). Indeed, three lines by the set of writing parameters were printed at different times and the measured widths did not present significant differences.

Finally, Fig. 10(c) highlights the limitations entailed by the use of a needle with an inner diameter of $110 \mu \mathrm{m}$. Such a needle cannot be used to print several sets of lines without observing a reduction of the lines widths. This phenomenon could be due to a partial clogging of the needle with silver micro-particles. However, this limitation can be overcome by using an ink with smaller silver particles, but this should not be assigned to the process itself.

Edges regularity. Fig. 11 shows the average standard deviation of the lines edges. Compared with the screen printing results, it appears that the developed process allowed producing lines that were more regular since the standard deviation was not higher than $10 \mu \mathrm{m}$, whereas it could reach $17 \mu \mathrm{m}$ for the screen printed lines. The reproducibility was also improved, as shown by the similar value of standard deviation recorded for all the lines printed with the same conditions. Generally speaking, lines produced with 3D printing devices are more regular than screen printed ones whatever the lines orientation. 


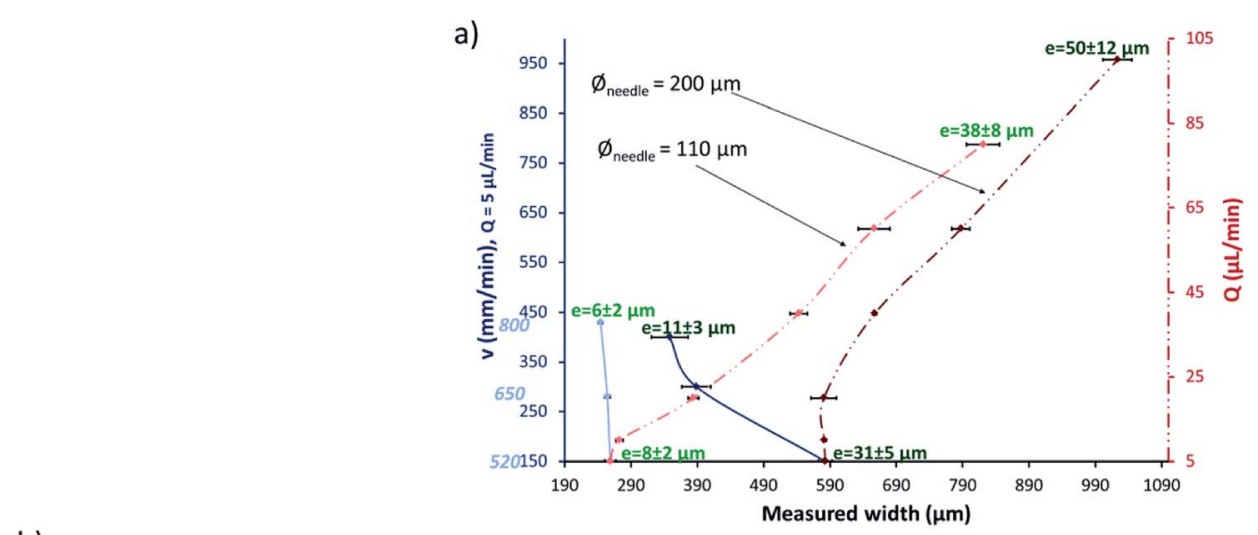

b)

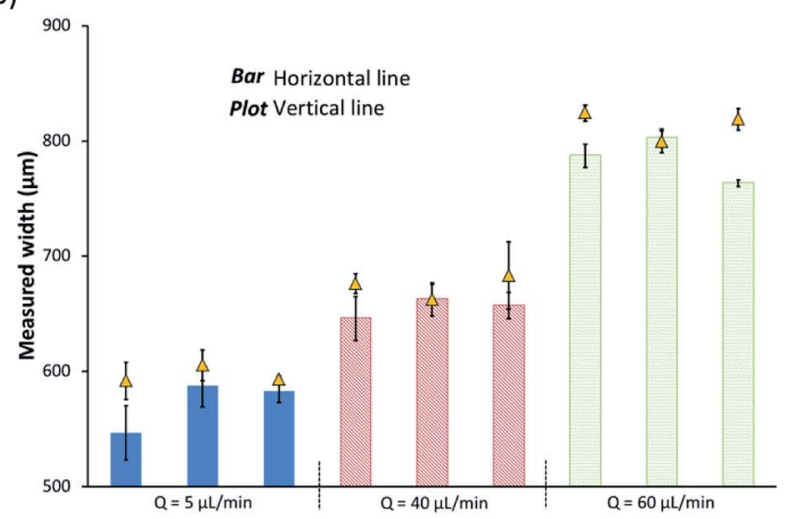

c)

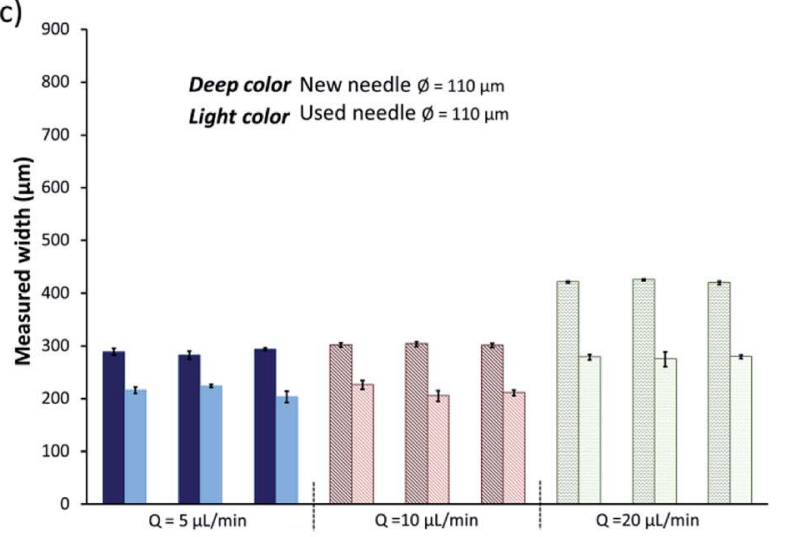

Fig. 10 Line widths according to the printing parameters: (a) horizontal lines width versus fluid flow, speed and inner needle diameter. The $e$ value is the measured thickness, (b) width comparison of horizontal and vertical lines printed with a needle of inner diameter $200 \mu \mathrm{m}$, (c) width comparison between several printings with the same $110 \mu \mathrm{m}$ inner diameter needle.

Here the highest amplitude of edges variation corresponded to $7 \%$ of the width of the thinnest lines represented in Fig. 11, graph (c), printed with a fluid flow rate of $5 \mu \mathrm{L} \min ^{-1}$. The highest amplitude recorded for screen printed lines corresponded to $12 \%$ of the line width.

By comparing graphs (a) and (b) with graph (c) in Fig. 11, it appears that the edges regularity is affected by the use of the needle with a small inner diameter. This can also be explained by an eventual partial clogging of the needle, which could be solved by using an ink with smaller particles.

Other characteristics. The roughness of lines was independent of the printing conditions and was about $1.4 \mu \mathrm{m} \pm 0.5 \mu \mathrm{m}$, twice the roughness of the screen printed lines. Such a difference may be due to the way the ink was deposited. During screen printing, there is an adhesion zone between the screen and the substrate during the squeeze stroke. The ink is therefore squeezed between these two elements. On the contrary, with the modified 3D printing device, the ink is deposited on the substrate without any contact between this latter and the needle. The ink can hence organize itself before drying.

Concerning the adhesion, the tape test led to the same result as the one obtained with screen printing. This property is actually mainly driven by the affinity between the ink and the substrate and the printing process does not impact this phenomenon.

Finally, the study of the lines printed with the 3D printing device showed that:
- A similar range of widths than the ones obtained with a standard screen printing process can be reached with the $3 \mathrm{D}$ written process. However, printing lines with a width inferior to $350 \mu \mathrm{m}$ requires a frequent change of needle. Nevertheless, this limitation can be overcome by selecting ink with smaller silver particles.

- The lines printed with the developed process have more regular edges than those produced by screen printing.

- The thickness of the lines is dependent on the deposited quantity. Large lines cannot present the same thickness than thinner lines.

- The line orientation does not affect the quality of the printed lines.

- The surface roughness of 3D-written lines is twice the roughness of the screen printed ones.

Electrical performances of the printed lines. After drying, the resistivity $\rho$ of lines printed by screen printing and by the developed direct writing technique was calculated and compared to the specification given in the ink technical data sheet. The results are summarized in Fig. 12.

According to Fig. 12, the resistivity of the lines seems to be dependent on the line thickness. As has been demonstrated before, the thickness of lines printed with the developed 3D printing technique is dependent on the printing parameters. Therefore large lines dried at $110^{\circ} \mathrm{C}$, owning a high thickness, had a lower electrical performance than fine lines with a low thickness. This could be explained by a partial drying of the ink 

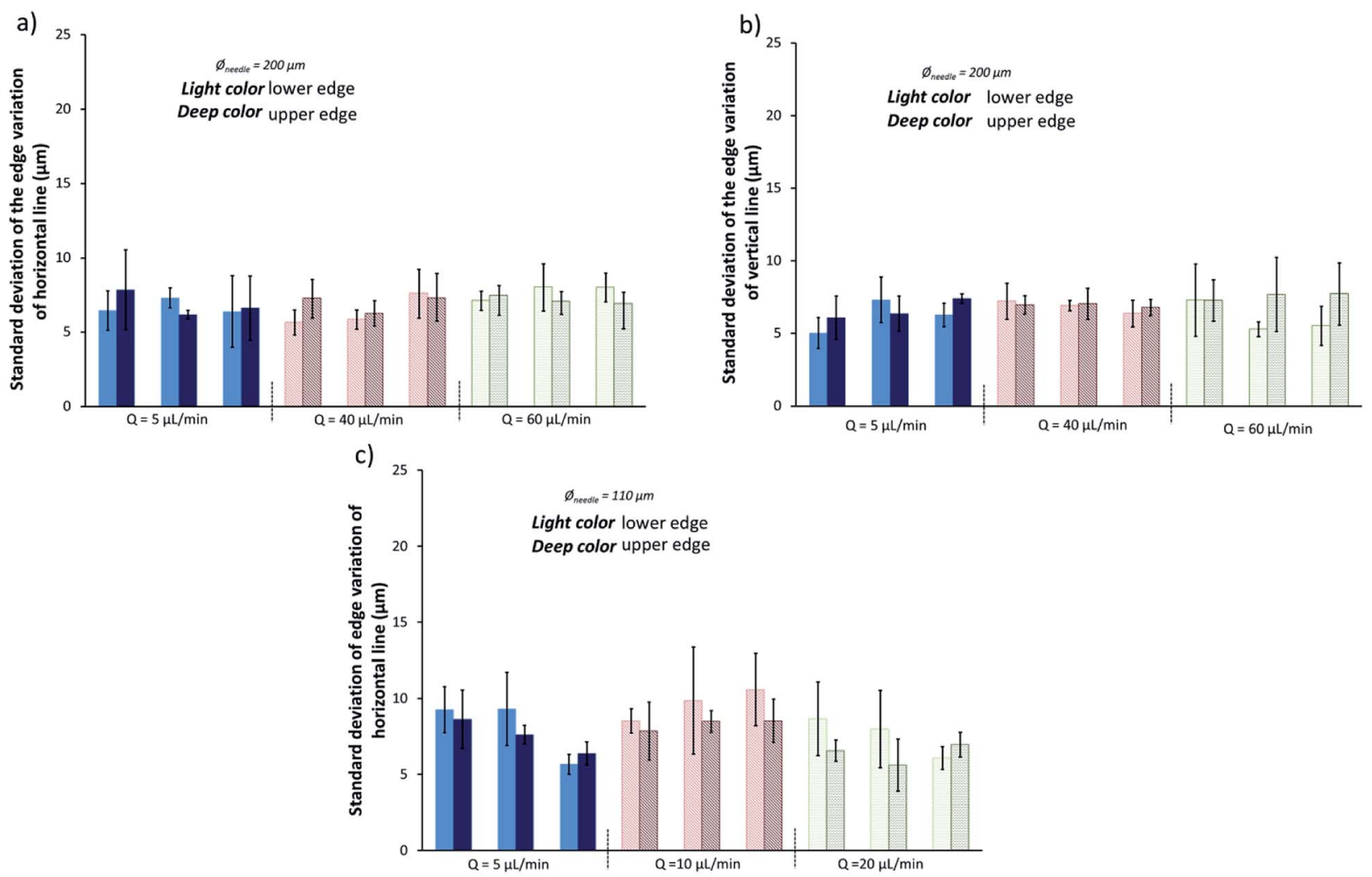

Fig. 11 Average standard deviation of the variation of lower and upper edges of lines printed with the modified 3D printing process: (a) 3 horizontal lines/condition printed with a $200 \mu \mathrm{m}$ inner diameter needle, (b) 3 vertical lines/condition printed with a $200 \mu \mathrm{m}$ inner diameter needle, (c) 3 horizontal lines printed with a $110 \mu \mathrm{m}$ inner diameter needle.

above a given thickness value. However, this limitation cannot be assigned to the direct writing method itself without studying the impact of the curing process and its optimisation. This point is the topic of another study.

Despite the previous observation, whatever the characteristics of the printed line, its resistivity was at least 1.5 time inferior to the expected value according to the ink' technical data sheet. Both studied printing techniques combined with a thermal curing at $110{ }^{\circ} \mathrm{C}$ are therefore compatible with electrical applications needing performances in accordance with the specifications of the conductive ink used in this study.

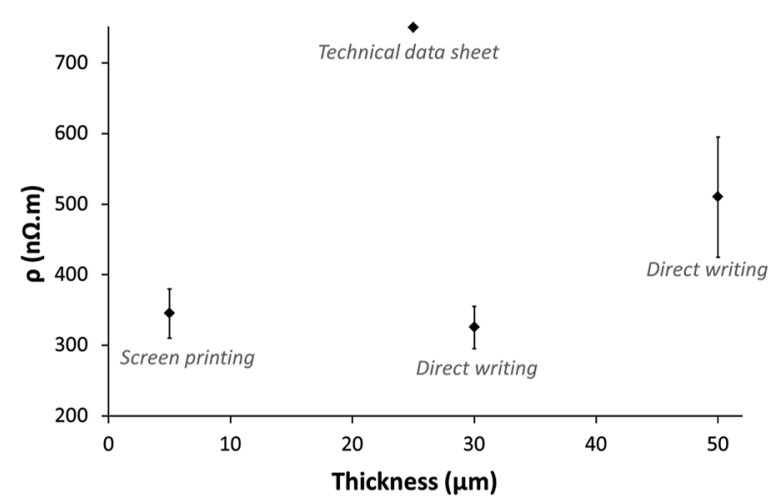

Fig. 12 Average resistivity of screen printed and direct written lines according to their thickness, and comparison with the data of the technical data sheet of the ink.

\section{Printing on 3D objects}

The developed device constituted by an eccentric screw dispenser and a Cartesian 3D printer allowed printing lines with a similar quality to ones obtained with a conventional screen printing system. Since the goal of the present study was to develop a process that could allow printing conductive lines on $3 \mathrm{D}$ objects, a few proof of concept systems were realized and tested (Fig. 13).

Three objects with different shapes were used as model substrates:

- Three coloured $\mathrm{PC}+\mathrm{ABS}$ items containing $90^{\circ}$ converging surfaces (on the top of the picture). The objective was to demonstrate the ability to print a continuous line along two perpendicular planes.

- A curved PC + ABS surface (on the bottom left of the picture). The direct writing permitted depositing a continuous line following the curve of the surface.

- An ABS hemisphere (on the bottom right of the picture) allowing verifying the writing on a spherical surface.

The conductivity of printed tracks was assessed by two means. First, the series resistance of the printed tracks was measured on the coloured items and on the curved one. The resistance value was about $7 \mathrm{Ohm}(300 \mathrm{n} \Omega \mathrm{m})$. When considering an antenna pattern with $L \times w \times e$ dimensions of about $400 \times 0.58 \times 0.028 \mathrm{~mm}^{3}$. Second, when a source was approached to the printed RFID receptor, the provided power 


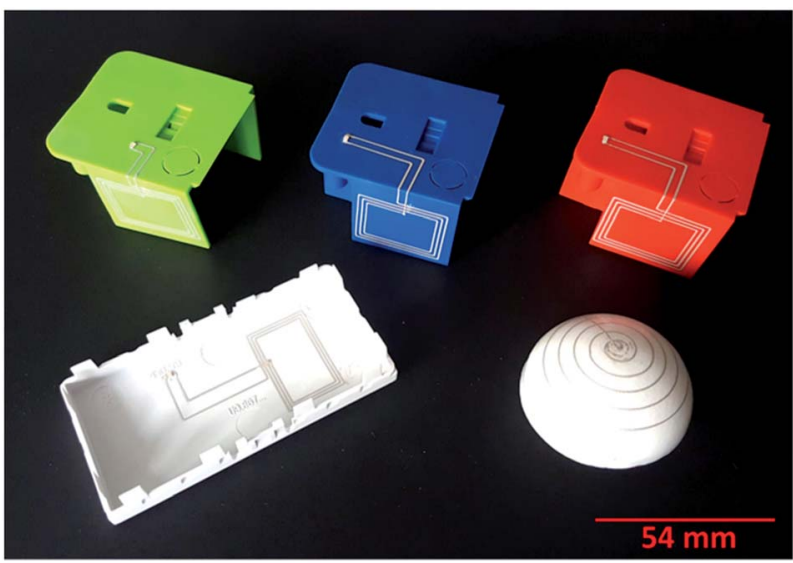

Fig. 13 Examples of prints of Henkel ink on thermoplastic 3D objects thanks to the developed device.

entailed a switching of the LED connected to the printed tracks, showing straightforwardly the correct behaviour of the 3D printed circuit.

\section{Conclusion}

A direct writing printing device was set up and evaluated by comparing the quality of the printed lines with the ones obtained by a standard screen printing procedure.

First, the study demonstrated the following key points:

(1) The direct writing process is compatible with commercial screen printing inks since the shear thinning behaviour of the latter facilitates the deposition step.

(2) The deposition ability of conductive patterns to adhere on to the thermoplastic substrates usually used in industry was confirmed.

(3) The direct writing method permitted printing patterns with a similar quality to the standard screen printing process. Some improvements were observed since line dribbles, typical of screen printing, were not observed. The printing quality was not sensitive to the pattern orientation.

(4) The conductivity of direct written lines after a drying in oven at $110{ }^{\circ} \mathrm{C}$ during $90 \mathrm{~min}$ was better than the announced value in the technical data sheet. The writing process could be used to produce electronical functions for which the ink specifications were sufficient. Moreover, that result could be improved by optimizing the ohmic curing. This is the topic of an ongoing study.

Second, two other observations deserve to be highlighted:

(1) The direct writing process permits saving ink. Indeed, even if the lines thickness is higher than the one of screen printed lines, only the required quantity is delivered by the volumetric dispenser while, in screen printing, a part of the ink remains on the screen meshes after the squeegee stroke.

(2) Some developments of the process are under further consideration. First, the printing speed can be easily improved by considering a more robust frame to support the printing head. Second, a greater degree of freedom can be envisaged for the printing head. Third, as advanced screen printing has been developed to be able to print ultrafine lines, a study on the ink properties and the printing parameters may cause the deposition of ultrafine lines on 3D objects.

Taking into account all these considerations and the demonstration of the printing on $3 \mathrm{D}$ objects, the $3 \mathrm{D}$ printing solution investigated can be seen as a viable alternative to the already available MID production techniques. For today, this satisfies the required quality to implement some electronic functions, such as antenna, in daily objects. In the near future, the developments under consideration will permit integrating fine and complex circuitry in any $3 \mathrm{D}$ object.

\section{Conflicts of interest}

There are no conflicts of interest to declare.

\section{Acknowledgements}

This work was made within the industrial chair MINT supported by the partnership Foundation of Grenoble INP and for which Schneider Electric is a sponsor. The study was realised at the LGP2 laboratory. LGP2 is part of the LabEx Tec 21 (Investissements d'Avenir - grant agreement no. ANR-11-LABX-0030) and of PolyNat Carnot Institute (Investissements d'Avenir grant agreement no. ANR-16-CARN-0025-01). This research was made possible thanks to the facilities of the TekLiCell platform funded by the Région Rhône-Alpes (ERDF: European regional development fund).

\section{Notes and references}

1 J. Gubbi, R. Buyya, S. Marusic and M. Palaniswami, Future Gener. Comput. Syst., 2013, 29, 1645-1660.

2 W. Xu, Adv. Mech. Eng., 2012, 2, 10.

3 N. Heininger, W. John and H.-J. Bossler, in International Congress MID, 2004.

4 C. Goth and M. Romer, Position sensor for adaptive speed control, (accessed May 19, 2017).

5 A. Islam, H. N. Hansen and N. Giannekas, CIRP Ann. Manuf. Technol., 2015, 64, 539-544.

6 C. Goth and M. Romer, Optics carrier and heating element for the diagnosis of caries, (accessed May 19, 2017).

7 F. Sonnerat, R. Pilard, F. Gianesello, D. Gloria, F. Le Pennec, C. Person, P. Brachat and C. Luxey, in 2013 IEEE Antennas and Propagation Society International Symposium (APSURSI), IEEE, 2013, pp. 2217-2218.

8 N. Heininger, T. P. Mansikkamäki, M. Kivikoski and I. Y.-H. Lee, in Proceedings of International Symposium on Antennas and Propagation, Korea, 2005.

9 A. Housden and J. Gould, Moulded Interconnect Devices, Prime Faraday Partnership, 2002.

10 F. Jorg, Three Dimensional Molded Interconnected devices (3D MID), Hanser Gardner Publications, 2014.

11 M. Pfeffer, C. Goth, D. Craiovan and J. Franke, in 2011 IEEE International Symposium on Assembly and Manufacturing (ISAM), IEEE, 2011, pp. 1-6. 
12 M. Ahmadloo and P. Mousavi, in 2013 IEEE MTT-S International Microwave Symposium Digest (IMS), IEEE, 2013, pp. 1-3.

13 C. Mariotti, M. M. Tentzeris and L. Roselli, in Microwave Symposium (MMS), 2015 IEEE 15th Mediterranean, IEEE, 2015, pp. 1-4.

14 F. A. H. Kim, S. B. H. Yun, I. Lee and others, in 2015 15th International Conference on Control, Automation and Systems (ICCAS), IEEE, 2015, pp. 1964-1968.

15 P. K. Wright, D. A. Dornfeld, A. Chen, C. C. Ho and J. W. Evans, Trans. NAMRISME, 2010, 38, 555-561.

16 N. Arnal, T. Ketterl, Y. Vega, J. Stratton, C. Perkowski, P. Deffenbaugh, K. Church and T. Weller, IEEE MTT-S International Microwave Symposium in 2015, IEEE, 2015, pp. 1-4.

17 B. Y. Ahn, D. J. Lorang and J. A. Lewis, Nanoscale, 2011, 3, 2700.

18 R. Ludwig, N.-C. Lee, S. R. Marongelli, S. Porcari and S. Chhabra, Proc. Nepcon West 99 Conf., Technical Session, 1999, vol. 13, pp. 576-588.

19 S. Thibert, J. Jourdan, B. Bechevet, D. Chaussy, N. ReverdyBruas and D. Beneventi, Mater. Sci. Semicond. Process., 2014, 27, 790-799.

20 F. Hoeng, A. Denneulin, N. Reverdy-Bruas, G. Krosnicki and J. Bras, Appl. Surf. Sci., 2017, 394, 160-168.

21 M. Neidert, W. Zhang, D. Zhang and A. Kipka, in Photovoltaic Specialists Conference, 2008. PVSC'08. 33rd IEEE, IEEE, 2008, pp. 1-4.
22 R. P. Chhabra and J. F. Richardson, Non-Newtonian flow in the process industries: fundamentals and engineering applications, Butterworth-Heinemann, 1999.

23 R. Faddoul, N. Reverdy-Bruas and A. Blayo, Mater. Sci. Eng., B, 2012, 177, 1053-1066.

24 P. Hahne, E. Hirth, I. E. Reis, K. Schwichtenberg, W. Richtering, F. M. Horn and U. Eggenweiler, Sol. Energy Mater. Sol. Cells, 2001, 65, 399-407.

25 C. P. Hsu, R. H. Guo, C. C. Hua, C.-L. Shih, W.-T. Chen and T.-I. Chang, J. Polym. Res., 2013, 20(277), 1-8.

26 H.-W. Lin, C.-P. Chang, W.-H. Hwu and M.-D. Ger, J. Mater. Process. Technol., 2008, 197, 284-291.

27 J. Hoornstra, A. W. Weeber, H. H. De Moor and W. C. Sinke, The importance of paste rheology in improving fine line, thick film screen printing of front side metallization, Netherlands Energy Research Foundation ECN, 1997.

28 M. Aoki, K. Nakamura, T. Tachibana, I. Sumita, H. Hayashi, H. Asada and Y. Ohshita, $30 \mu \mathrm{m}$ fine line printing for solar cells, IEEE Photovoltaic Specialists Conference, Florida, 2013, pp. 2162-2166.

29 R. Soukup, A. Hamáček and J. Řeboun, in 2012 35th International Spring Seminar on Electronics Technology (ISSE), IEEE, 2012, pp. 19-24.

30 D. Erath, A. Filipović, M. Retzlaff, A. K. Goetz, F. Clement, D. Biro and R. Preu, Sol. Energy Mater. Sol. Cells, 2010, 94, 57-61.

31 W. J. Hyun, E. B. Secor, M. C. Hersam, C. D. Frisbie and L. F. Francis, Adv. Mater., 2015, 27, 109-115. 